\title{
A Pelvic Muscle Precontraction Can Reduce Cough-Related Urine Loss in Selected Women with Mild SUI
}

\author{
Janis M. Miller, PhD, "James A. Ashton-Miller, PhD, ${ }^{\dagger}$ and John O. L. DeLancey, $M D^{\ddagger}$
}

\begin{abstract}
OBJECTIVES: To test the hypothesis that selected older women with mild-to-moderate stress urinary incontinence (SUI) can learn to demonstrate significantly reduced urine loss in 1 week by intentionally contracting the pelvic floor muscles before and during a cough (a skill we have termed "The Knack").
\end{abstract}

DESIGN: A prospective, randomized, single-blind interventional study.

SETTING: The Older American Independence Center, a federally sponsored research program affiliated with the University of Michigan in Ann Arbor, Michigan.

PARTICIPANTS: Twenty-seven women with a mean (SD) age of $68.0(5.5)$ years, self-reported SUI, and demonstrable urine loss during a deep cough.

INTERVENTION: Women were randomized to an immediate intervention group (Group I: $n=13$ ) who were taught the Knack after their first clinic visit, or a wait-listed control group (Group II: $n=14$ ) who were taught the Knack after 1 month.

MEASUREMENTS: At 1 week after instruction, we tested the efficacy of the Knack in a standing stress test by (1) comparing the volumes of cough-related urine loss leaked by all subjects, with and without use of the Knack, and (2) comparing the volumes of cough-related urine loss leaked by Group I, using the Knack, with Group II, which had not yet been taught the Knack.

RESULTS: Intra-individual results showed that at 1-week follow-up, the Knack was used to reduce urine loss resulting from a medium cough by an average of $98.2 \%$, compared with that of a similar cough performed 1 minute before without the Knack $(P=.009)$; likewise urine loss was reduced by an average of $73.3 \%(P=.003)$ in a deep cough. Reduction in urine loss was not significantly correlated with a digital measure of pelvic floor muscle strength.

CONCLUSION: Within 1 week, selected older women with

From the "Institute of Gerontology, tDepartment of Mechanical Engineering and Applied Mechanics, Department of Biomedical Enginecring, and Institute of Gerontology; and $¥ D$ epartment of Obstetrics and Gynecology, University of Michigan, Ann Arbor, Michigan.

This work was supported by Public Health Service Grants P30 AG 08808, R01 DK 47516, and T32 AG 001140.

Presented, in part, at the annual meeting of the International Continence Society in Athens, Greece, August, 1996.

Address correspondence to Janis M. Miller, PhD, GG Brown Laboratorics, Rm 3208, University of Michigan, $A n n$ Arbor, MI 48109-2125. Address reprint requests to James A. Ashton-Miller, $\mathrm{PhD}$, at the same address. mild-to-moderate SUI can acquire the skill of using a properly-timed pelvic floor muscle contraction to significantly reduce urine leakage during a cough. J Am Geriatr Soc $46: 870-874,1998$.

Stress urinary incontinence (SUI) is the involuntary loss of urine during coughing, sneezing, laughing, or other physical activities that increase intra-abdominal pressure. ${ }^{1}$ Conservative treatments for SUI often begin with the prescription of 2 to 3 months of repetitive strengthening, or Kegel, ${ }^{2}$ exercises for the pelvic floor muscles. Despite outcome measures showing up to $44 \%$ improvements in pelvic floor muscle strength ${ }^{3}$ and from 31 to $73 \%$ reduction in self-reported leakage episodes, ${ }^{4}$ in the absence of causal evidence, it is not clear how much, if any, of the latter improvement can be ascribed to the pelvic floor muscle strengthening.

Some practitioners teach patients with mild SUI to use a pelvic floor muscle contraction to prevent stress-related urine loss as part of their exercise intervention aimed at strengthening the pelvic floor muscles. For example, Burgio ${ }^{5}$ prescribed daily pelvic floor muscle exercise and "Patients were trained to actively use these muscles during physical stress. In the clinic they practiced contracting the muscles immediately before and during voluntary coughing."

We (JM and JOLD) have also observed that certain women instructed in pelvic muscle strengthening exercises report a reduction in stress-related urine leakage within as little as 1 to 2 days. Because pelvic floor muscle hypertrophy cannot have occurred over such a short time frame, ${ }^{6,7}$ we posited that these women had taught themselves a new skill similar to that advocated by Burgio: namely, to time a contraction of their pelvic floor muscles intentionally with the moment they expect to leak urine so as to prevent that urine loss. The skill of intentionally contracting the pelvic floor muscles just before and throughout the intra-abdominal pressure rise associated with a stressful activity such as a cough, in order to prevent urine loss, is a skill we have termed "The Knack." We distinguish this use of a single, intentionally timed contraction from the widely used repetitive (Kegel) exercises intended to strengthen the pelvic floor (see Discussion).

The purpose of this study, therefore, was to test the following hypothesis: Selected older women with SUI, able to demonstrate the ability to isolate and contract their pelvic floor muscles, can be taught to use the Knack to demonstrate significantly reduced cough-related urine leakage when com- 
pared with (1) leakage in similar coughs performed without the Knack or (2) leakage in untreated controls not yet taught the Knack.

\section{METHODS}

Twenty-seven community-dwelling women of mixed parity with mild-to-moderate SUI were assigned randomly, in blocks of two, to either an immediate intervention group (Group I: $\mathbf{n}=13$ ) or a wait-listed control group (Group II: $n=14)$. The age range of the subjects was from 60 to 84 years, with a mean (SD) of 68.4 (5.5) years. The majority $(83 \%)$ was parous, with a mean (SD) parity of $2.8(2.1)$. Inclusion criteria included age 60 years and older, selfreported symptoms of SUI and frequency of leakage occurring at least weekly and up to 5 times/day as measured on a 6-day self-report urination diary, ${ }^{8}$ and direct visualization of urine loss during a cough. Women were excluded if they had a history of systemic neuromuscular disease, previous bladder surgery, or an active urinary tract infection. Women were also excluded for leakage that was delayed after coughing and categorized as detrusor instability ( 2 women), leakage that saturated a paper towel and/or pooled on the floor when coughing in the standing posture ( 2 women), inability to demonstrate any voluntary contraction of the pelvic floor muscles despite detailed instruction during the pelvic exam (3 women), and significant coexistent pelvic organ prolapse below the hymenal ring ( 2 women).

Written consent for the intervention was obtained before baseline measures were taken. At the first clinic visit, women in Group I were taught about the basic physiological and functional properties of the pelvic floor muscles as well as instructed, with vaginal palpation, on how to contract the pelvic floor muscles. The instruction first included digital confirmation that correct volitional control of pelvic floor muscle contraction was acquired without an observable Valsalva maneuver. Each woman was then taught to hold the pelvic floor muscle contraction while breathing in and out. They were then asked to practice the Knack by contracting the pelvic floor muscles "just before initiating a hard cough and maintain the contraction throughout the cough."

Instruction was given to hold the contraction until after the abdominal wall had relaxed post-cough. Subjects were asked to practice the Knack at home and to return to the clinic for testing at the 1-week follow-up visit. Measures gathered at baseline and at the 1-week follow-up included the self-report 6-day urination diary, ${ }^{8}$ a digital test for pelvic muscle strength ${ }^{9-11}$ (possible score range: $0-21$ ) whose validity has been reported elsewhere, ${ }^{11,12}$ and a standing stress test described below.

During the month that Group II participants were waitlisted, the patients in that group underwent an additional baseline measurement to serve as untreated controls for comparison with Group I at its 1-week follow-up time. After their 1-month wait, however, Group II received the same intervention and 1-week follow-up testing as Group 1 . Thus, in another test of the primary hypothesis, the leakage volumes with and without the Knack were collected at 1-week follow-up in all 27 patients and compared directly using paired tests.

We measured the volume of urine leaked during a standing stress test using a quantitative method, the Paper Towel Test, whose sensitivity and reliability have been demonstrated elsewhere. ${ }^{13}$ During testing, wetness from any previ- ous measurement and from possible vaginal secretions were eliminated by having the woman dry the perineum thoroughly before each measure. Total leakage onto a single 26-cm-wide by 9-cm-long towel (Item \#219-24; Handifold Towels, Fort Howard Corporation, Green Bay, WI) held against the perineum was measured after three serial medium coughs ("cough at about half your maximal strength"), each cough being performed at a comfortable interval with a separate breath. Using a fresh paper towel, total leakage was also measured after three deep coughs ("cough as hard as you can"), each again performed with a separate breath. Once Knack instruction was provided, the 1-week follow-up visit included repeating the above Paper Towel Test protocol in the following order: (1) medium cough $\times 3$ with Knack, (2) medium cough $\times 3$ without Knack, (3) deep cough $\times 3$ without Knack, (4) deep cough $\times 3$ with Knack. Before Knack instruction, subjects simply performed the two sets of medium and deep coughs without the Knack. Within 10 seconds of finishing a test, the resultant orthogonal diameters of the wetted area were measured and its area calculated using the formula for an ellipse. The estimated volume loss was then found from a calibration curve based on a second order polynomial regression (coefficient of determination $=$ .99) derived from measuring the wetted areas resulting from titrating a minimum of $0.05 \mathrm{~mL}$ (equivalent to 1 drop) to a maximum of $6 \mathrm{~mL}$ of water onto 30 different towels. The maximum volume of $6 \mathrm{~mL}$ was chosen to avoid inaccuracies caused by towel saturation. (The calibration curve was such that a linear regression fit with a slope of $0.039 \mathrm{~mL} / \mathrm{cm}^{2}$ and coefficient of determination $=.97$ ). The test-retest repeatability of the Paper Towel Test is known to be acceptable inasmuch as $95 \%$ of the root-mean-square within- and acrossvisit differences were less than $1 \mathrm{~mL} .{ }^{13}$

The volume of urine in the bladder was not controlled other than asking the women to come to the appointment with a subjectively "full bladder." Inclusion criteria for this study, however, required that women had to void at least 100 $\mathrm{mL}$ urine at the end of their visit as proof that their bladder had contained at least that volume during the tests.

Descriptive statistics were used to analyze demographic and categorical variables. The primary hypothesis was tested at 1-week follow-up using a within-subjects comparison by applying the paired Student's $t$ test to the logarithm of the Paper Towel Test leakage volume measured with and without the use of the Knack. Similary, to check for cohort effects, an independent $t$ test comparing logarithms of Group I leakage change with the Knack with Group II leakage change without the Knack was also performed using the Group I 1-week follow-up time point. Pearson correlation was used to test the secondary hypothesis that reduction in urine loss was proportional to pelvic muscle strength. We used two-sided tests in all comparisons, and a value of $P<.05$ was considered statistically significant.

\section{RESULTS}

Across all subjects, mean (SD) self-reported leakage frequency on the 6-day diary was 1.36 (1.39) episodes/day, ranging from 0 to 5 episodes/day. All 27 subjects reported leakage with coughing by questionnaire. Postinstructional digital pelvic floor muscle strength ranged from 4 to 15 points, with a mean (SD) of 11.0 (4.6) points, with digital strength scores in Groups I and II of 11.2 (4.3) and 10.8 (4.9) points, respectively. Because no significant age, parity, 
strength, or leakage frequency differences were found between Groups I and II, their data were pooled for the first test of the primary hypothesis, the results of which are described below.

\section{Intraindividual Analyses One Week After Leaming the Knack ( $n=27$ subjects)}

The primary hypothesis was supported: use of the Knack reduced leakage on the medium cough Paper Towel Test from $19.9(36.7) \mathrm{cm}^{2}$ to $0.4(1.1) \mathrm{cm}^{2}$ a reduction of $98.2 \%$ $\left(P=.009\right.$, on a paired difference of $\left.18.7(35.5) \mathrm{cm}^{2}\right)$. Similarly, use of the Knack on the deep cough Paper Towel Test reduced leakage from $29.8(36.16)$ to $8.0(18.2) \mathrm{cm}^{2}$, a reduction of $73.3 \%(P=.003$, on a paired difference of 21.9 $(34.0) \mathrm{cm}^{2}$ ) (Figure 1). Thus, using the conversion given in Methods, use of the Knack in a deep cough reduced leakage from an average of $1.41 \mathrm{~mL}$ to an average of $0.38 \mathrm{~mL}$ (Figure 1).

\section{Between-Groups Comparisons at the Group I One-Week Follow-Up Time Point}

Leakage on the medium cough Paper Towel Test was similar in both groups without Knack use (Table 1), but when Group I used the Knack, subjects leaked $98.1 \%$ less in the medium cough Paper Towel Test than did members of Group II, who had not yet learned the Knack $(P=.293$, Table 1$)$. Similarly, leakage in the deep cough Paper Towel Test was similar in both groups without Knack use (Table 1), but when Group I used the Knack they reduced their leakage significantly in a deep cough Paper Towel Test by $79.1 \%$ when compared with the untreated controls $(P=.031$, Table 1$)$. These data again support the primary hypothesis that the Group I reduction in deep cough leakage was attributable to learning the Knack and suggest that the improvement was neither caused by a cohort effect nor by mere contact with the investigators.

In a test of the secondary hypothesis, digital test scores of pelvic floor muscle strength (Table 2) did not correlate with urine loss reduction for either medium $(r=0.02, P=.936)$ or deep coughs $(r=0.29, P=.138)$. No significant difference in pelvic floor muscle strength was found between the two visits in either group.

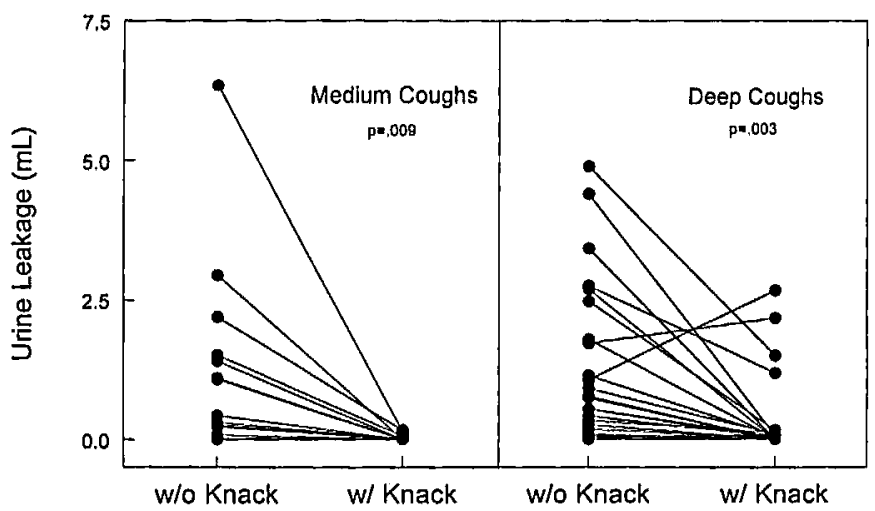

Figure 1.The effect of the Knack on the total volume of urine leaked in three separate medium coughs (left) and three separate deep coughs (right) at 1-week follow-up. Each line joins total leakage in each of the 27 women observed without the Knack (denoted by "w/o Knack") with that observed with using the Knack (denoted "w/ Knack").
For the medium and deep coughs, most, but not all, subjects experienced improvement in Paper Towel Test leakage using the Knack (Figure 1). Examination of individual outcomes (Table 2 ) revealed that 25 of the 27 women leaked urine on at least one of the two sets of deep coughs. Twenty of those 25 women $(80 \%)$ were able to use the Knack to reduce leakage on a deep cough, eight women could reduce leakage to a drop or two, and a further five were able to completely prevent any urine loss. More than half (16 of 27) of the women leaked urine on one of the medium coughs. Of those 16 women, $15(94 \%)$ were successful in using the Knack to reduce their urine leakage, and the last woman's negligible leakage was unaltered. The mean voided volume at the end of the clinic visit for these subjects was 249 (121) mL, thus establishing the average minimum volume of urine in the bladder during these tests.

\section{DISCUSSION}

This paper demonstrates for the first time that selected older women with SUI learned to use the Knack in less than a week to reduce the volume of urine leaked during a cough. In one-sixth of these older women, complete prevention of urine loss during a deep cough was obtained in that time frame, whereas half of the remaining women reduced their leakage to less than $1 / 10 \mathrm{~mL}$ (or 2 drops). Since the aim was to have the pelvic floor muscle contraction developed fully before the onset of the rise in intraabdominal pressure associated with the cough, we taught women to contract the pelvic floor muscles about 1 second in advance of the cough. We did this because, as shown earlier, nearly half a second is required to volitionally develop maximal tension in striated muscle. ${ }^{14}$ No increase in strength was found within the 1 -week study duration, and thus the results support the contention made in the Introduction that it was the acquisition of the skill of properly timing the pelvic floor muscle contraction, rather than the absolute strength of the pelvic floor muscles, that was important in reducing cough-related SUI in this study.

A caveat is in order. These results were obtained during a clinic visit. It is one thing for a subject to demonstrate a learned behavior in the clinic. It is quite another to go home, learn to anticipate leakage on a particular activity, and remember to use the Knack every time it is needed in spite of the distractions of every day life. Such a change would require both behavior modification and practice in the same way that it takes encouragement and time to develop the habit of covering one's mouth before coughing. A test of how well women with SUI can incorporate the Knack into preventing urine loss in their daily activities was beyond the scope of the present study, but is currently underway in our unit.

There is logic in using an intervention that involves the Knack, actively using the pelvic floor muscles to prevent stress-related urine loss. It is well known that in healthy women, a volitional pelvic floor muscle contraction can, in seconds, develop sufficient urethral closure pressure to halt urine flow during micturition. Secondly, Enhorning has demonstrated that some women naturally increase their intraurethral closure pressure by some 30 to $40 \mathrm{~cm} \mathrm{H}_{2} 0100$ to 300 ms before a cough. ${ }^{15}$ This behavior, since corroborated by Constantinou and Govan, ${ }^{16}$ has been ascribed to anticipatory voluntary or reflex contraction of the pelvic floor muscles. ${ }^{15}$ Thirdly, there is a biomechanical basis for the expectation that a volitional pelvic muscle contraction can increase urethral closure pressure. Contributions to this increase can 
Table 1. Effect of Knack on Mean (SD) Area* $\left(\mathrm{cm}^{2}\right)$ of Urine Leaked in Consecutive Series of Three Coughs of Given Intensity at 1-Weck Follow-up, by Group

\begin{tabular}{|c|c|c|c|c|}
\hline \multirow[b]{2}{*}{ Group } & \multicolumn{2}{|c|}{ Medium Cough } & \multicolumn{2}{|c|}{ Deep Cough } \\
\hline & With Knack & Without Knack & Without Knack & With Knack \\
\hline Group I $(n=13)$ & $0.4(1.04)^{\ddagger}$ & $23.0(44.6)$ & 32.7 (33.9) & $5.4(15.3)^{\S}$ \\
\hline Group $\|(n=14)$ & $\begin{array}{c}\text { Without Knack } 1^{q l} \\
21.2(44.8)^{\dagger \neq}\end{array}$ & $\begin{array}{c}\text { Without Knack } 2^{\pi} \\
23.8(46.5)\end{array}$ & $\begin{array}{c}\text { Without Knack } 1 \\
30.4(44.2)\end{array}$ & $\begin{array}{c}\text { Without Knack } 2 \\
26.8(46.7)^{\dagger \S}\end{array}$ \\
\hline
\end{tabular}

${ }^{*} 1 \mathrm{~cm}^{2}$ is equivalent to $0.039 \mathrm{~mL}$ (see Methods)

tenotes based on data from $\mathrm{n}=10$ only due to missing dara.

$F_{\text {denotes }} \mathrm{P}=.293$

Sdenotes $P=.031$

I 1 and 2 refer to the first and sccond three-coughs series in these controls (who had not yet learned the Knack).

Table 2. Areas of Wetness $\left(\mathrm{cm}^{2}\right)$ on the Paper Towel Test 1-Week Post-Knack Instruction. Baseline Pclvic Floor Muscle (PFM) Digital Strength Scores and Voided Volumes Are Also Given

\begin{tabular}{|c|c|c|c|c|c|c|}
\hline \multirow[b]{2}{*}{ Subject ID } & \multicolumn{2}{|c|}{ Medium Cough } & \multicolumn{2}{|c|}{ Deep Cough } & \multirow{2}{*}{$\begin{array}{c}\text { PFM } \\
\text { Strength } \\
\text { Score }\end{array}$} & \multirow[b]{2}{*}{$\begin{array}{c}\text { Voided } \\
\text { Volume }(\mathrm{mL})\end{array}$} \\
\hline & $\begin{array}{l}\text { Without } \\
\text { Knack }\end{array}$ & $\begin{array}{l}\text { With } \\
\text { Knack }\end{array}$ & $\begin{array}{l}\text { Without } \\
\text { Knack }\end{array}$ & $\begin{array}{r}\text { With } \\
\text { Knack }\end{array}$ & & \\
\hline 1 & 75.4 & 0.0 & 125.6 & 38.5 & 12 & 133 \\
\hline 2 & 0.0 & 0.0 & 19.2 & 0.0 & 7 & 163 \\
\hline 3 & 11.0 & 0.0 & 11.0 & 0.8 & 17 & 285 \\
\hline 4 & 0.4 & 0.0 & 1.2 & 1.6 & 4 & 237 \\
\hline 5 & 0.0 & 0.0 & 8.8 & 2.9 & 18 & 240 \\
\hline 6 & 27.5 & 0.0 & 14.1 & 0.1 & 8 & 141 \\
\hline 7 & 28.0 & 0.0 & 29.4 & 1.6 & 17 & 297 \\
\hline 8 & 75.4 & 0.0 & 27.4 & 68.7 & 11 & 433 \\
\hline 9 & 7.9 & 0.0 & 87.9 & 0.1 & 9 & 123 \\
\hline 10 & 38.5 & 1.6 & 23.6 & 2.4 & 11 & 117 \\
\hline 11 & 0.4 & 0.4 & $0.0^{\dagger}$ & $0.0^{\dagger}$ & 4 & 170 \\
\hline 12 & 0.0 & 0.0 & 0.0 & 0.0 & 9 & 240 \\
\hline 13 & 0.0 & 0.0 & 14.1 & 1.5 & 17 & 236 \\
\hline 14 & 0.0 & 0.0 & 69.1 & 0.0 & 13 & 139 \\
\hline 15 & 56.1 & 4.3 & 70.7 & 30.6 & 6 & 596 \\
\hline 16 & 0.0 & 0.0 & 2.4 & 0.0 & 10 & 521 \\
\hline 17 & 2.0 & 0.0 & 6.9 & 0.0 & 19 & 244 \\
\hline 18 & 162.5 & 3.5 & 44.0 & 55.9 & 11 & 300 \\
\hline 19 & 0.0 & 0.0 & 113.0 & 1.8 & 17 & 169 \\
\hline 20 & 35.7 & 0.0 & 63.6 & 4.3 & 15 & 130 \\
\hline 21 & 0.4 & 0.0 & 4.7 & 0.6 & 8 & 100 \\
\hline 22 & 0.0 & 0.0 & 45.9 & 0.6 & 13 & 290 \\
\hline 23 & 5.9 & 0.0 & 0.0 & 0.0 & 8 & 238 \\
\hline 24 & 0.0 & 0.0 & 0.0 & 0.8 & 7 & 174 \\
\hline 25 & 0.0 & 0.0 & 0.8 & 2.0 & 6 & 158 \\
\hline 26 & 0.0 & 0.0 & 0.0 & 0.0 & 6 & 200 \\
\hline 27 & 6.3 & 0.0 & 20.0 & 0.0 & 15 & 360 \\
\hline
\end{tabular}

Indicates non-zero leakage between 0 and $0.04 \mathrm{~cm}^{2}$, the resolution of the method

come from the volitional recruitment of the striated muscle of the urogenital sphincter as well as elevation and stiffening of the hammock-like layer of passive tissues under the midurethra by the intact sub-urethral attachments ${ }^{17}$ of the volitionally recruited pelvic floor muscles.

We are aware of only one study ${ }^{5}$ that explicitly describes instructing patients to time a pelvic floor muscle contraction to reduce urine loss and that was used, together with muscle strengthening, to reduce urine leakage during activities expected to cause SUI. The outcome measure that was reported, however, evaluated change in incontinence episodes across time, rather than change in activity-specific urine loss as a result of a properly timed pelvic floor muscle contraction. One other publication contains a general discussive statement 
that "women with SUI were encouraged to use pelvic floor muscle contractions to control involuntary urine loss." 18 Neither study, however, quantified how much a single pelvic floor muscle contraction can be used to reduce urine loss during a specified activity known to cause SUI. Our results support the wisdom of teaching selected women with SUI the skill of using a single pelvic muscle contraction to prevent anticipated activity-related urine loss in conservative behavioral interventions. ${ }^{5}$

Recognizing that correct use of a voluntary pelvic floor muscle contraction to prevent leakage during a stressful activity is an acquired motor skill, namely that the pelvic floor muscle contraction must be fully developed before and during a rise in abdominal pressure, one of us (JAAM) called the preventative maneuver "the Knack" because the word "knack" means a task that requires dexterity or a clever way of doing something. By using the term Knack, we imply the skilled use of a single pelvic floor muscle contraction to prevent anticipated involuntary urine loss.

The Knack was shown to reduce leakage whether it was performed with the first (as in the medium cough) or the second (as in the deep cough) of a pair of coughs, suggesting order effects were minimal. We did not measure abdominal pressure during the coughs because we did not want to affect the continence system function, geometry, or pelvic floor muscle function using an invasive transducer. However, it is unlikely that our results can be explained on the basis of a diminished abdominal pressure every time the Knack was used because we have found no significant differences in cough-related maximum vesical pressure between deep coughs performed with and without the Knack. ${ }^{19}$ Another methodological limitation was the lack of standardization of bladder volume below $400 \mathrm{~mL} \cdot{ }^{20}$ However, the voided volumes demonstrated that the bladder contained at least $100 \mathrm{~mL}$ urine during testing, with an average of more than 2.5 times that volume.

It is possible that in some women, improvement in pelvic floor muscle motor control skill and/or contraction strength could bring further improvement in Knack efficacy. The minimum pelvic floor muscle strength required for an effective Knack remains unknown, but it likely depends on striated urethral muscle capacity as indicated by urethral resting closure pressure, the achievable volitional increase in that closure pressure, the pressure transmission to the urethra, ${ }^{21}$ and the magnitude of the abdominal pressure rise. Pragmatically speaking, if the Knack does not reduce cough-related leakage to $1 \mathrm{~mL}$ or less per leakage episode, the practical implication is that the intervention is of minimal therapeutic value and should be supplemented by other forms of therapy. In this study, we have demonstrated the efficacy of the Knack in older women with mild SUI; there is reason to believe that the Knack would be as, or possibly more, effective in younger women given the additional continence margin ${ }^{19}$ afforded by their higher resting urethral closure pressures. ${ }^{21}$

Use of the Knack clearly requires certain cognitive capabilities; women with dementia would be unlikely to benefit from its use, for example. It is also unlikely to benefit women who are unable to link leakage with a specific activity, but simply "find themselves wet." Even for cognitively intact women, the skill acquisition can require several clinic visits before the pelvic muscles can be properly identified, differentiated from other nearby muscles, and brought under reliable control. $^{2,5}$ We were interested to find that some women found the immediate positive feedback gained from seeing the Knack reduce the wetted area on the Paper Towel Test a revelation, a veritable "Ah $\mathrm{Ha}$ " experience. Use of the Knack will not benefit women whose SUI has reached the point that they leak on repetitive activities, such as walking or jogging, because of the difficulty of maintaining a pelvic floor muscle contraction for more than a few seconds. We wish to reemphasize that prescription of the Knack would be illogical in women who are unable to demonstrate that they can use a pelvic floor muscle contraction volitionally to raise their intraurethral closure pressure because of neurological, connective tissue, or motor control impairments.

With proper selection criteria, the simplicity and effectiveness of the Knack make it a promising first-stage self-help intervention for women with mild, stress-related leakage who are able to perform a pelvic floor muscle contraction.

\section{ACKNOWLEDGMENTS}

The authors gratefully acknowledge the assistance of Lisa Carchidi, RN, and Kathy Krajewski, BS.

\section{REFERENCES}

1. Fantl JA, Newman DK, Colling J ct al. Urinary Incontinence in Adults: Acute and Chronic Management. Clinical Practice Guideline, No. 2, 1996 Update. Rockville, MD: U.S. Department of Health and Human Services. Public Health Service, Agency for Health Care Policy and Research. AHCPR Publication No. 96-0682. March 1996.

2. Kegel A. Progressive resistance exercise in the functional restoration of the perineal muscles. Am J Obstet Gynecol 1948;56:238 -248.

3. Ferguson KL, McKey PL, Bishop KR et al. Stress urinary incontinence: Effect of pelvic muscle exercise. Obstet Gynecol 1990;75:671-675.

4. Wells TJ. Pelvic (floor) muscle exercise. J Am Geriatr Soc 1990;38:333-337.

5. Burgio K, Robinson J, Engel B. The role of biofeedback in Kegel exercise training for stress urinary incontinence. Am J Obstet Gynecol 1986;154:5864.

6. Ikai M, Fukunaga T. A study on training effects on strength per unit of cross- sectional area of muscle by means of ultrasonic measurement. Int $\mathrm{Z}$ Angew Physiol 1970;28:173-180.

7. Fiaterone MA, Marks EC, Ryan ND et al. High intensity strength training in non octogenarians. JAMA 1990;263:3029-3024.

8. Wyman JF, Choi S, Harkins $S$ et al. The urination diary in evaluation of incontinent women: A test-retest analysis. Obstct Gynecol 1988;71:812-817.

9. Miller JM. On Pelvic Floor Muscle Function and Stress Urinary Incontinence: Effects of Posture, Parity, and Volitional Control [doctoral thesis]. School of Nursing, University of Michigan, Ann Arbor, MI, 1996.

10. Brink C, Sampselle C, Wells TJ et al. A digital test for pelvic muscle strength in older women with urinary incontinence. Nurs Res 1989;38:196-199.

11. Sampselle C, Brink C, Wells TJ. Digital measurement of pelvic muscle strength in childbearing women. Nurs Res 1989;38:134-138.

12. Brink C, Wells TJ, Sampselle C. A digital test for pelvic muscle strength in women with stress incontinence. Nurs Res 1994;43:352-356.

13. Miller JM, Ashton-Miller JA, DeLancey JOL. Quantification of coughrelated urine loss using the paper towel test. Obstet Gynecol 1998;91:705709.

14. Thelen DG, Schultz AB, Alexander NB, Ashton-Miller JA. Effects of age on rapid ankle torque development. J Gerontol 1996;51A:M226-232.

15. Enhorning $G$. Simultaneous recording of the intravesical and intraurethral pressure. Acta Obstet Gynecol Scand 1961;276(suppl):1-69.

16. Constantinou $\mathrm{CE}$, Govan DE. Spatial distribution and timing of transmitted and reflexly generated urethral pressure in healthy women. J Urol $1982 ; 127: 964-969$

17. DeLancey JOL. Functional anatomy of the female lower urinary tract and pelvic floor. In: Neurobiology of Incontinence (Ciba Foundation Symposium 151). Chichester: Wiley, 1990, pp 57-76.

18. Dougherty M, Bishop K, Mooney R et al. Graded pelvic muscle exercise; Effect on stress urinary incontinence, J Reprod Med 1993;38:684-691.

19. Kim K, Ashton-Miller JA, Schultz $A B$ et al. The vesico-urethral pressuregram analysis of urethral function under stress. J Biomech 1996;30:19-25.

20. Miklos JR, Sze EH, Karram MM. A critical appraisal of the measuring leakpoint pressures in women with stress incontinence. Obstet Gynecol $1995 ; 86: 349-352$.

21. Rud 'T. Urethral pressure profile in continent women from childhood to old agc. Acta Obstet Gynecol Scand 1980;59:331-335. 